\title{
Morphometric Analysis of the Mandible of Tuj and Morkaraman Sheep
}

\section{Yasin Demiraslan ${ }^{1 * *}$ Filiz Gülbaz ${ }^{2}$ Sami Özcan ${ }^{1}$ Mustafa Orhun Dayan $^{3}$ Yalçın Akbulut ${ }^{4}$}

\footnotetext{
${ }^{1}$ Department of Anatomy, Faculty of Veterinary Medicine, Kafkas University, 36100 Kars -TURKEY

${ }^{2}$ Ataturk Vocational School of Health Services, Kafkas University, 36100 Kars - TURKEY ${ }^{3}$ Department of Anatomy, Faculty of Veterinary Medicine, Selcuk University, 42100 Konya -TURKEY

${ }^{4}$ Kars Collage of Health, Kafkas University, 36100 Kars -TURKEY
}

\section{Abstract}

In the present study, it was aimed to determine morphometric differences of the mandible of Tuj and Morkaraman sheep having widespread breeding area in the Eastern Anatolia Region. In this study, 20 mandibles of the male Tuj and Morkaraman sheep were used. The 16lenghts taken to the mandible were measured by electronic digital calliper. Furthermore, after the mandible were photographed and printed to milimetric paper, the 4-angles studied were measured with gonimeter. The results of this study proved that the means of the length and angle measurements obtained from Morkaraman sheep were mostly higher than Tuj sheep. The angle of margo ventralis mandibulae (A4) and the mandible height at the level of the rear alveolar edge with 3rd molar tooth (L14) had a significant statistical difference $(p<0.05)$. According to the correlation values, it was seen that $A 2$ values (the angle between the proc. condylaris and ramus mandibulae) of the both sheep breeds were in negative relation with the other mandible measurements. As a result, the obtained data indicate that genetic doesn't significantly affect the morphometry of the mandible of the Morkaraman and Tuj sheeps.

Keywords: Mandibula, Morkaraman sheep, Morphometry, Tuj sheep

\section{Introduction}

In Turkey, Morkaraman sheep, which is the dominant race of East- 
ern Anatolia Region has numerically in the second rank. Morkaraman sheep is also grown in Iran with name of Kızıl or Gızıl. Tuj sheep breeding in North East Anatolia Region belongs to potential of $0.03 \%$ in among the sheep races found in Turkey (Akcapınar, 2000; Kaya, 2001; Ozcan, 1990). Tuj sheep is known as Tuchin in Caucasia Region (Akcapınar, 2000; Ozcan, 1990).

The morphometry is a method of which description and statistical analysis of shape variation is in among samples of organisms. The topics of the morphometry have a wide scale including geographic localities, developmental stages, genetic and environmental effects. The morphometry allows making statistical analysis as numerical or graphical measurements of the length between specific two points or angles (Rohlf and Marcus, 1993).

Some studies have been previously carried out on the mandible morphometry by using different methods in the varied animal species (Alpak, 2003; Ince Gezer and Pazvant, 2010; Olopade and Onwuka, 2005; Onar et al., 1999; Yalcın et al., 2007). Ozcan et al. (2010) has been studied about with skull of Morkaraman and Tuj sheeps species. Moreover, mandibles of the Akkaraman and Anatolia Wild have been compared using the geomet- rical morphometry (Yalcın et al., 2010). The morphometric aspects of the mandible of Mehraban sheep (Karimi et al., 2012), sheep and roe deer (Avdic et al., 2013) have been analysed. However, in the literature, knowledge related to mandible morphometry of Morkaraman and Tuj sheeps was insufficient. For this reason, in the study it was aimed to determine the mophometric differences of the mandible of Morkaraman and Tuj sheep widespreadly found in East Anatolia Region of Turkey.

\section{Material and Methods}

In this study 20 mandibles of male Tuj and Morkaraman (aged 10-12months) obtained from Kars Municipality Slaugterhouse were used. Weight of animals averaged $32 \pm 1.8$ $\mathrm{kg}$. The mandibles were separated from the skulls, and their skin and muscles were removed. After the mandibles were macerated for 2030 minutes with hydrogen peroxide minutes, the 16 differences measurements were by using electronic (0.00, BTS, UK) from the mandibles. These measurements were given in following and at the Fig. (2).

Length 1(L1): lenght between gonion caudale and infradentale.

Length 2(L2): length between infradentale and aboral edge of condylar process. 
Length 3(L3): length between gonion caudale and aboral alveolar edge of $3^{\text {rd }}$ molar tooth.

Length 4(L4): length between infradentale and aboral alveolar edge of $3^{\text {rd }}$ molar tooth.

Length 5(L5): length between gonion caudale and rostral alveolar edge of $2^{\text {nd }}$ premolar tooth.

Length 6(L6): length between gonion caudale and aboral edge of mental foramen.

Length $7(L 7)$ : length between first premolar tooth and last molar tooth.

Length 8(L8): length between first and last molar teeth.

Length $9(L 9)$ : length between first and last premolar teeth.

Length 10(L10): length of diestema.

Length 11(L11): length between gonion ventrale and condylion.

Length 12(L12): length between gonion ventrale and the deepest point of incisura mandibulae

Length 13(L13): length between gonion ventrale and coronion.

Length 14(L14): height of mandible level of alveolar edge of $3^{\text {rd }}$ molar tooth.

Length 15(L15): height of mandible level of rostral alveolar edge of $1^{\text {st }}$ molar tooth.

Length 16(U16): height of mandible level of rostral alveolar edge of $2^{\text {nd }}$ premolar tooth.

The terms used in the osteometric measurements;
Condylion (Cnd): The end point of caudal of the processus condylaris Coronion (Cr): The end point of cadal of the processus coronoideus Gonion ventrale $(G v)$ : The end point of the ventral border of the angulus mandibulae

Gonion caudale (Gc): The end point of the caudal border of the ramus mandibulae

Infradentale (Id): The rostro-dorsal point of alveoli dentalis between mandibular incisive teeth.

The length measurements of the mandible were shown in Fig(1). The obtained length values were standardized with the length and heights of the mandibles because the animals used in this study were in different ages and live weights. These ratios were determined as L2/L1, L3/L1, L4/L1, L5/L1, L6/L1, L7/L1, L8/L1, L9/L1, L10/L1, L11/L3, L12/L3, L14/L3, L15/L3 and L16/L3.

For measuring the angles, the mandibles were photographed and printed to graph paper. Then after, the four angles were measured with goniometer. These measurements were given in Fig (2).

Angle 1 (A1): angle between margo ventralis mandibulae and margo caudalis mandibulae.

Angle 2 (A2): angle between tuber mandibulae of the condylar process and margo caudalis mandibulae. 
Angle 3 (A3): angle between ramus mandibulae and margo alveolaris of pars molaris corporis mandibulae.

Angle 4 (A4): angle made between rostral edge of the corpus mandibulae and the margo ventralis mandibulae.

In this study, the method of Driesch (1976), based on osteometric length measurements were adopted (Fig 1), while Onar et al., (1999) method, based on angle measurements were followed (fig 2).

Statistical analysis of the study was conducted by using Statistical Package for Social Sciences (SPSS) version 17.0 software package. Continuous variables were stated as arithmetical mean \pm standard deviation. A Mann-Whitney $U$ test was used to compare the data. Moreover, the correlation of the values obtained from this study was analyzed (Pearson's correlation test). Nomina Anatomica Veterinaria (2005) based for terms used in the study.

\section{Results and Discussion}

The findings of this study were shown in Tables $(1,2,3,4)$.

Ketani and Sagsoz (2009) reported that the genetic variation is affected during the development of the mandible. In this study, the mandibles of the Tuj and Morkaraman breeds had been compared by the morphometric methods. It has been revealed that the genetic variation between the two sheep breeds was expressed by the methods used. Thereby, osteometric and angular measurements among the breeds have been evaluated as statistically and probable differences have been identified. In the study, it has been determined that averages of measurements of the length and angle obtained from Morkaraman sheep have been higher than Tuj sheep. However, it has been investigated that the statistical difference has been in the values of the L14 and A4 $(p<0.05)$. Moreover, the difference of the L14 height in the level of molar teeth of Morkaraman sheeps' mandible is higher than Tuj Sheep's mandible, and this height was statistically significant $(p<0.05)$. It is shown that the difference of the A4 values of Tuj Sheep's mandible was more parallel to the ground than Morkaramans' sheep mandible and this parallelism is statistically meaningful $(p<0.05)$.

According to the correlation analysis. Although there was a strong positive and significant correlation among the values of the L2 and L9 in Morkaraman sheep $(p<0.01)$, there was a weak positive and statistically unimportant correlation among the same measurements of Tuj sheep $(p>0.05)$. This value 
proved increase or decrease of the length between condylion and infradentale in Morkaraman sheep is statistically related to the length of the premolar teeth. While there was a strong positive and significant correlation between the values of the L8 and L9 in Morkaraman sheep $(p<0.01)$, there was a weak negative and statistically unimportant correlation in Tuj sheep ( $p>0.05)$.

The lengths of the premolar and molar teeth in Morkaraman sheep are linked.

The results of this study proved that the negative correlation was strong and statistically important among of the values of the L14, L15 and L3 in the Morkaraman sheep. In Tuj sheep, a negative, strong and statistically significant correlation has been also detected (except for L15 and L3).

According to the correlation values of the L16 and A2, The parallelism to ground of ramus mandibulae has increased because of the increase in the height of the mandible at the level of the last molar tooth in Morkaraman sheep.

Avdic et al. (2013), mentioned in sheep and roe deer, the values of the L1, L7, L10, L11, L13 and L15 as follows respectively $17.6,6.20$, $4.66,6.80,9.96,2.33 \mathrm{~cm}$ in sheep, and $15.6,6.33,4.55,5.85,8.43$, 1.55 in roe deer. In this study, the same values were determined re- spectively as $15.24,6.85,3.72$, 6.21, 8.7, $2.12 \mathrm{~cm}$ in Morkaraman sheep, and 14.78, 6.61, 3.64, 6.09, $8.54,2.06 \mathrm{~cm}$ in Tuj sheep.

Some morphometric values of Mehra-ban sheep, WAD(West African Dwarf) and Black Bengal goats compared with Morkaraman and Tuj sheep of this study are shown in the Table (4). Accordingly, it has been identified that the longest and highest mandibles respectively belong to Mehraban, Morkaraman and Tuj.

In the present study, the obtained length and height values of the mandibles were standardized because the animals used for research were in different ages and live weights. Accordingly it has been concluded that there is no statistically significant values between the sheep races.

In conclusion, it has been observed that the genetic factor has no influence on the morphometry of mandible in Morkaraman and Tuj sheeps. Although the useful morphometric differences such as the values of the correlation and indices have been presented in line with the conducted statistical analysis.

\section{References}

Akcapınar, H. (2000): Sheep farming. $2^{\text {nd }}$ ed., pp: 109-115. Ismat Publishing, Ankara. 
Alpak, H. (2003): Morphometry of the mandible of German Shepherd Dog (Alsatian) puppies using computed tomographic analysis. Isr. J. Vet. Med., 58 (1) 15-17.

Avdic, R., Hadziomerovic, N., Tandir, F., Pamela, B., Velida, C. (2013): Analysis of Morphometric Parameters of the Roe Deer Mandible (Capreolus Capreolus) and Mandible of the Sheep (Ovis Aries). Veterinaria, 62 (1-2), 1-9.

Ince Gezer, N., Pazvant, G. (2010): Morphometry of the Mandible in Rats (Wistar Albino). J. Fac. Vet. Med. Istanbul Univ., 36 (1), 51-56.

Karimi, I., Hadipour, M., Nikbakht, P., Motamedi, S. (2011): The Lower Jawbone of Mehraban Sheep: A Descriptive Morphometric Approach World's Vet. J., 2(4): 57-60.

Kaya, S. (2001): Effect of Early Weaning in the Tuj lambs on Growth Performance and Development Spesifications. Master Thesis. Health Sciences Institues of Kafkas Univ. Kars, Turkey.

Ketani, A.M., Sagsoz, H. (2009): Histomorphometrical evaluation of the Effects of Gender on the Mandibular Condyle in Rats. Atatürk Univ. Vet. Bil. Derg., 4 (1), 31-38.
Nomina Anatomica Veterinaria (2005): International Committee on Veterinary Gross Anatomical Nomenclature. 5th ed., Published by the Editorial Committee, Hannover.

Olopade, J.O., Onwuka, S.K. (2005): Some Aspects of the Clinical Anatomy of the Mandibular and Maxillofacial Regions of the West African Dwarf Goat in Nigeria. Int. J. Morphol.,23 (1), 33-36.

Onar, V., Kahvecioglu, O., Mutus, R., Alpak, H. (1999): Morphometric Analysis of the Mandible in German Shepherd Dogs. Tr. J. Vet. Anim. Sci., 23 (2), 329-334.

Ozcan, L. (1990): Sheep farming. Ministry of Agriculture, Forestry, and Rural Affairs, Publication Department, Ankara, s. 100-103, 113-114.

Ozcan, S., Aksoy, G., Kurtul, I., Aslan, K., Ozudogru, Z. (2009): A Comparative Morphometric Study on the Skull of the Tuj and Morkaraman Sheep. Kafkas Univ. Vet. Fak. Derg., 16 (1): 111-114.

Rohlf, F.J., Marcus, L.F. (1993): A revolution in morphometrics. Trends Ecol. Evol., 8, 129-132.

Von Den Driesch, A. (1976): A Guide to the measurement of animal bones from archaeological 
sites. pp. 31-34. Peabody Museum Bulletin I. Cambridge M.A., Harvard University.

Uddin, M.M., Uddin, Ahmed, S.S., Islam, K.N., Islam, M.M. (2009): Clinical anatomy of the head region of the Black Bengal goat in Bangladesh. Int. J. Morphol., 27(4):12691273.

Yalcin, H., Kaya, M.A., Arslan, A. (2010): Comparative Geometrical Morphometries on the Mandibles of Anatolian Wild Sheep (Ovis gmelini anatolica) and Akkaraman Sheep (Ovis aries). Kafkas Univ. Vet. Fak. Derg., 16 (1) 55-61.

Yalcin, H., Kayis, S.A., Arslan, A. (2007): A Comparative MacroAnatomic, Mechanical and Geometric Morphometrics Study on Tree and Ground Squirrel and Rat. Vet. Bil. Derg., 23 (1), 83-95.

\section{Corresponding author:}

Yasin Demiraslan,

Department of Anatomy, Faculty of Veterinary Medicine, Kafkas University, 36100 Kars -TURKEY,

Call: +90 0474242 6839/5308, Fax: +90 04742426853 ,

E-mail: yasindemiraslan@hotmail.com 
Analysis of the Tuj and Morkaraman Sheep's mandible

Demiraslan et al.,

Table (1): The osteometric and angle measurements of the mandibles of Tuj and Morkaraman sheep. The unit of the mean values and the standard deviations are $\mathrm{mm}$. * $p<0.05$

\begin{tabular}{|c|c|c|}
\hline Measurements & Tuj sheep & Morkaraman sheep \\
\hline L1 & $147.76 \pm 5.40$ & $152.43 \pm 7.47$ \\
\hline L2 & $155.59 \pm 5.28$ & $160.43 \pm 7.24$ \\
\hline L3 & $43.61 \pm 2.32$ & $43.20 \pm 4.56$ \\
\hline L4 & $107.86 \pm 4.04$ & $112.81 \pm 4.81$ \\
\hline L5 & $102.94 \pm 2.57$ & $106.97 \pm 6.24$ \\
\hline L6 & $118.85 \pm 2.52$ & $122.29 \pm 5.19$ \\
\hline L7 & $66.14 \pm 1.97$ & $68.51 \pm 2.70$ \\
\hline L8 & $50.80 \pm 1.85$ & $53.12 \pm 1.96$ \\
\hline L9 & $14.94 \pm 0.84$ & $15.14 \pm 0.90$ \\
\hline L10 & $36.44 \pm 2.5$ & $37.16 \pm 1.88$ \\
\hline L11 & $60.86 \pm 1.67$ & $62.08 \pm 4.13$ \\
\hline L12 & $55.06 \pm 2.26$ & $57.19 \pm 4.23$ \\
\hline L13 & $85.37 \pm 2.74$ & $86.97 \pm 6.08$ \\
\hline L14* & $36.86 \pm 0.53$ & $38.88 \pm 1.81$ \\
\hline L15 & $20.61 \pm 0.64$ & $21.18 \pm 0.55$ \\
\hline L16 & $15.89 \pm 0.86$ & $16.40 \pm 0.88$ \\
\hline A1 & $101.40 \pm 3.21$ & $103.40 \pm 3.19$ \\
\hline A2 & $78.60 \pm 3.21$ & $76.60 \pm 3.19$ \\
\hline A3 & $144.60 \pm 5.73$ & $148.30 \pm 3.62$ \\
\hline $\mathrm{A}^{*}{ }^{*}$ & $30.80 \pm 2.80$ & $39.20 \pm 5.66$ \\
\hline
\end{tabular}


Analysis of the Tuj and Morkaraman Sheep's mandible

Demiraslan et al.,

Table (2): The correlation values of the mandibles Tuj and Morkaraman sheeps. ' $\rightarrow$ ' The correlation values of Morkaraman sheep, ' $\downarrow$ ' The correlation values of Tuj sheep. * $p<$ $0.05,{ }^{* *} p<0.01$.

\begin{tabular}{|c|c|c|c|c|c|c|c|c|c|c|c|c|c|c|c|c|c|c|c|c|}
\hline 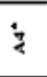 & ప్త్రీ & 행 & $\frac{3}{0}$ & $\frac{\bar{u}}{0}$ & त̂ & है & 离 & $\frac{\bar{\sigma}}{0}$ & $\frac{9}{3}$ & \begin{tabular}{|l|l} 
\\
5
\end{tabular} & สั & $\frac{a_{0}^{\prime}}{3}$ & $\frac{3}{3}$ & $\frac{\AA}{0}$ & 菏 & \begin{tabular}{|c|} 
\\
\end{tabular} & $\tilde{\tilde{c}}$ & $\frac{1}{2}$ & 8 & \\
\hline$z$ & $\frac{9}{0}$ & $\overline{\underline{p}}$ & 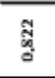 & 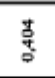 & 总 & के & $\begin{array}{ll} \\
\\
0 \\
0\end{array}$ & 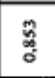 & 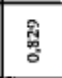 & 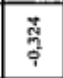 & 志 & 害 & 莡 & 范 & 熍 & $\frac{\tilde{\alpha}}{\frac{\alpha}{\sigma}}$ & 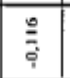 & $\stackrel{0}{3}$ & & है \\
\hline 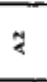 & $\begin{array}{l}\text { nे } \\
c\end{array}$ & జิ & 善 & 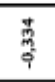 & 喜 & 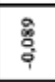 & 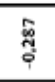 & 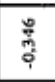 & : & \begin{tabular}{|l}
$\frac{0}{0}$ \\
\multirow{3}{*}{} \\
\end{tabular} & $\begin{array}{l}\stackrel{8}{0} \\
\end{array}$ & के & $\frac{n}{0}$ & 蛋 & 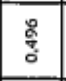 & 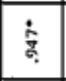 & . & & 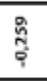 & $\frac{\hat{3}}{3}$ \\
\hline z & $\frac{n}{0}$ & : & 畨 & 农 & 悉 & 愛 & 歒 & 品 & 悉 & \begin{tabular}{|l}
$\frac{0}{0}$ \\
\end{tabular} & \begin{tabular}{|l}
8 \\
\end{tabular} & 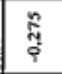 & 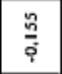 & 沽 & \begin{tabular}{|l|}
\multirow{2}{*}{} \\
\multirow{i}{*}{} \\
\end{tabular} & 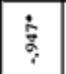 & & .⿳亠二口犬. & बू. & : \\
\hline$\stackrel{0}{\Xi}$ & 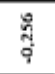 & 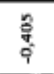 & $\frac{8}{0}$ & $\begin{array}{l}\overrightarrow{T_{1}} \\
\dot{a}\end{array}$ & $\frac{n}{a}$ & 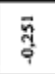 & 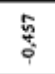 & 产 & \begin{tabular}{|l|l}
$\frac{0}{6}$ \\
\end{tabular} & $=$ & $E$ & 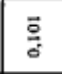 & 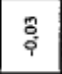 & 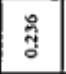 & \begin{tabular}{|l|}
$a_{0}^{\circ}$ \\
\end{tabular} & & \begin{tabular}{|l|}
\multirow{z}{*}{} \\
co \\
\end{tabular} & 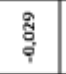 & 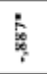 & ఫ్ \\
\hline$\frac{n}{2}$ & है & $\frac{n}{\partial}$ & $\dot{\dot{m}}$ & 啇 & : & 总 & 총 & ส্ণ & 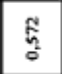 & 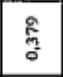 & $\begin{array}{l}\text { E } \\
\end{array}$ & 葛 & : & $\dot{\overline{\bar{c}}}$ & & 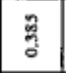 & $\begin{array}{l}0 \\
\\
\end{array}$ & $\frac{9}{\partial}$ & 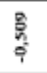 & สุ๊ \\
\hline$\Xi$ & ¿ & 厗 & 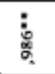 & 幾 & : & 8 & जे & 高 & 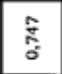 & : & $\frac{1}{6}$ & $\dot{\bar{\partial}}$ & 蜜 & & \% & 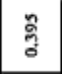 & है & $\begin{array}{l}\vec{z} \\
a_{1}\end{array}$ & $\frac{\hbar}{b}$ & 总 \\
\hline 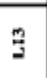 & : & $\dot{\phi}$ & $\dot{q}$ & $\frac{q}{2}$ & 6 & 商 & ईई. & 葛 & के & $\omega_{\infty}$ & \begin{tabular}{|l|}
$\vdots$ \\
$\vdots$ \\
\end{tabular} & 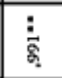 & & 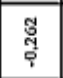 & 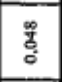 & : & \begin{tabular}{|l|}
$\frac{q}{0}$ \\
$\begin{array}{c}0 \\
\end{array}$ \\
\end{tabular} & 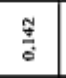 & 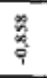 & $\frac{n}{3}$ \\
\hline$\cong$ & ¿ே. & $\frac{2}{3}$ & $\dot{\tilde{\sigma}}$ & 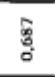 & \% & 㩊 & $\begin{array}{l}0 \\
\text { है } \\
0\end{array}$ & 总 & 莕 & 古 & 产 & & $\mathscr{E}_{0}$ & 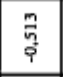 & 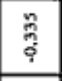 & 萦 & 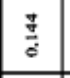 & 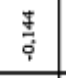 & 等 & $\bar{o}$ \\
\hline$\Xi$ & $\dot{s}$ & $\dot{\phi}$ & : & 感 & : & $\frac{\dot{0}}{\partial}$ & 奱 & $\stackrel{\circ}{\frac{0}{0}}$ & : & 4 & & $=$ & $\begin{array}{l}0 \\
\text { त. } \\
0\end{array}$ & $\frac{\Phi}{\frac{m}{0}}$ & त् & $\begin{array}{l}\vec{\nabla} \\
0 \\
\end{array}$ & శ్ & శ్శ్ & 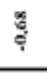 & 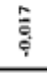 \\
\hline$\stackrel{\circ}{\beth}$ & $\begin{array}{l} \\
0 \\
0 \\
0\end{array}$ & $\begin{array}{l}9 \\
9 \\
0\end{array}$ & $\frac{\pi}{6}$ & $\mathbb{Z}$ & $\frac{8}{\delta}$ & $\overrightarrow{w_{0}^{2}}$ & בे & 蒙 & สั้ & & \begin{tabular}{|l|}
0 \\
$\vdots$ \\
$\vdots$ \\
\end{tabular} & 蹇 & 总 & 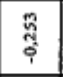 & 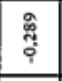 & $\begin{array}{l}\text { Te } \\
\text { R. } \\
\end{array}$ & \begin{tabular}{|l|}
$\frac{n}{0}$ \\
\end{tabular} & $\begin{array}{l}\frac{n}{0} \\
\end{array}$ & 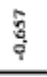 & జू \\
\hline s & : & $\overline{\dot{\alpha}}$ & $\begin{array}{l}0 \\
0 \\
\end{array}$ & $\bar{z}$ & $\begin{array}{l}\vdots \\
\vdots \\
\vdots\end{array}$ & $\frac{\vdots}{\vdots}$ & $\dot{8}$ & 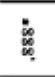 & & 迨 & $\begin{array}{ll}5 \\
\end{array}$ & 9 & 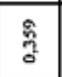 & 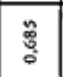 & $\frac{\dot{a}}{\partial}$ & $\begin{array}{l}0 \\
\text { 艿 } \\
\end{array}$ & \begin{tabular}{|l|}
$\frac{2}{2}$ \\
$\frac{2}{0}$ \\
1
\end{tabular} & $\frac{m}{0}$ & $\begin{array}{l}\infty \\
\\
\end{array}$ & 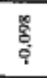 \\
\hline$\$$ & $\begin{array}{l}\mathbf{W} \\
\text { 䓌 }\end{array}$ & $\overline{\bar{i}}$ & $\begin{array}{l} \\
\\
0\end{array}$ & $\begin{array}{l}0 \\
0 \\
0\end{array}$ & 参 & 营 & $\dot{\hat{g}}$ & & 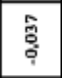 & 亭 & 范 & 范 & 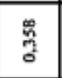 & 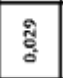 & $\ddot{i}$ & $\begin{array}{l}\frac{2}{0} \\
\\
\end{array}$ & \begin{tabular}{|l|}
$\frac{2}{3}$ \\
0 \\
0
\end{tabular} & 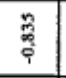 & 荌 & $\begin{array}{l}0 \\
0 \\
0\end{array}$ \\
\hline$=$ & $\dot{\dot{t}}$ & ¿ें & $\stackrel{n}{0}$ & $\dot{\dot{\delta}}$ & $\dot{\bar{\alpha}}$ & is & & हैं & 离 & 8 & $\therefore$ & $\begin{array}{ll} \\
0\end{array}$ & न्च & $\begin{array}{l}m_{0} \\
\\
\end{array}$ & : & 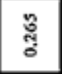 & 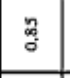 & $\begin{array}{l}0 \\
0 \\
0\end{array}$ & $\stackrel{\circ}{\stackrel{0}{i}}$ & $\stackrel{\infty}{0}$ \\
\hline 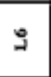 & 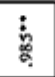 & $\dot{\delta}$ & శ్ & $\dot{8}$ & 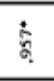 & & $\dot{s}$ & $\dot{\mathrm{g}}$ & 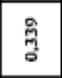 & 喽 & 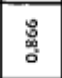 & . & $\begin{array}{l}0 \\
0 \\
0 \\
0 \\
0\end{array}$ & $\because$ & 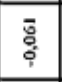 & $:$ & \begin{tabular}{|l|}
$\frac{0}{0}$ \\
\\
\end{tabular} & \begin{tabular}{l|}
0 \\
$\vdots$ \\
$\vdots$ \\
$\vdots$
\end{tabular} & 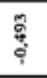 & \begin{tabular}{l}
\multirow{2}{*}{} \\
\end{tabular} \\
\hline 3 & $\begin{array}{l} \\
\\
\end{array}$ & s. & दूँ & $\begin{array}{l}0 \\
\end{array}$ & & $\dot{\vdots}$ & 苦 & 䬭 & $\begin{array}{l}0 \\
\vdots \\
0\end{array}$ & $\begin{array}{l}\text { 孰 } \\
\end{array}$ & 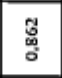 & ఫุ & $\begin{array}{l}\frac{n}{2} \\
\dot{\sigma}\end{array}$ & 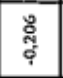 & \begin{tabular}{|l|}
$\frac{9}{0}$ \\
1
\end{tabular} & $\overline{8}$ & \begin{tabular}{|l|}
\multirow{2}{*}{} \\
\end{tabular} & 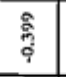 & क्ष & $\frac{m}{0}$ \\
\hline 3 & $\overrightarrow{\hat{x}}$ & 宙 & कू & & 亳 & $\dot{\bar{a}}$ & : & 30 & 종 & 0 & $0^{\circ}$ & 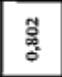 & 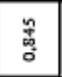 & 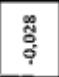 & $\begin{array}{l}\frac{9}{3} \\
6\end{array}$ & $\begin{array}{l}\mathrm{m} \\
\mathrm{s} \\
0\end{array}$ & \begin{tabular}{|l|}
8 \\
8 \\
\end{tabular} & $\begin{array}{l} \\
\\
\dot{p} \\
\end{array}$ & 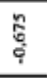 & : \\
\hline 3 & $\overrightarrow{\mathrm{s}}$ & : & & 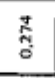 & : & $\frac{\pi}{0}$ & 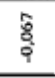 & ने & 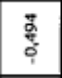 & 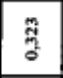 & 0 & $\begin{array}{l}0 \\
0 \\
0 \\
0\end{array}$ & 豪 & के & \begin{tabular}{|l|}
\multirow{3}{0}{} \\
$\vdots$ \\
\end{tabular} & \begin{tabular}{|l|} 
磍 \\
3
\end{tabular} & $\frac{2}{9}$ & $\frac{\pi}{0}$ & 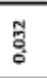 & $\begin{array}{l}\frac{8}{8} \\
\frac{1}{4}\end{array}$ \\
\hline$I$ & $\begin{array}{l} \\
\vdots \\
\%\end{array}$ & & $\bar{E}$ & $\dot{\vdots}$ & $\begin{array}{l} \\
8 \\
8\end{array}$ & ذे. & t: & בֶّ & $\frac{E}{0}$ & 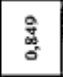 & $\begin{array}{ll}\frac{\pi}{2} \\
0 \\
0\end{array}$ & 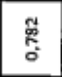 & 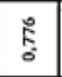 & $\begin{array}{l}0 \\
0 \\
0 \\
0\end{array}$ & 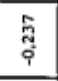 & : & $\overrightarrow{\text { ริ }}$ & $\overline{3}$ & $\begin{array}{l}5 \\
\vdots \\
\vdots\end{array}$ & $\ddot{z}$ \\
\hline 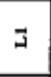 & & ڤั่ & $\frac{0}{8}$ & $\begin{array}{l}\dot{\alpha} \\
\dot{\alpha}\end{array}$ & $\dot{0}$ & $\dot{8}$ & $\begin{array}{l}0.0 \\
8 \\
0\end{array}$ & 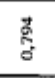 & $\frac{6}{0}$ & 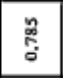 & $\begin{array}{l}\text { \% } \\
\text { \% } \\
\end{array}$ & 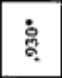 & 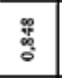 & đ̊̃ & 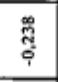 & 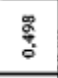 & $\begin{array}{l}: \\
\\
\end{array}$ & $\stackrel{\circ}{\grave{c}}$ & $\begin{array}{l} \\
\vdots \\
\vdots \\
\end{array}$ & $\frac{\delta}{8}$ \\
\hline i. & $=$ & $z$ & 3 & 3 & 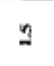 & 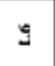 & $=$ & 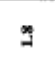 & 9 & $\mathbf{z}$ & 3 & $\Xi$ & $\frac{2}{3}$ & 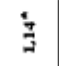 & $\lambda$ & $\bar{z}$ & $=$ & ₹ & $<$ & $\dot{z}$ \\
\hline
\end{tabular}


Analysis of the Tuj and Morkaraman Sheep's mandible

Demiraslan et al.,

Table (3): The standardization data of the mandibles of Tuj and Morkaraman sheeps.

\begin{tabular}{|c|c|c|}
\hline Rates & Morkaraman sheep & Tuj sheep \\
\hline L2/L1 & $1.05 \pm 0.02$ & $1.05 \pm 0.01$ \\
\hline L3/L1 & $0.30 \pm 0.01$ & $0.28 \pm 0.02$ \\
\hline L4/L1 & $0.73 \pm 0.01$ & $0.74 \pm 0.02$ \\
\hline L5/L1 & $0.70 \pm 0.01$ & $0.70 \pm 0.01$ \\
\hline L6/L1 & $0.81 \pm 0.02$ & $0.80 \pm 0.01$ \\
\hline L7/L1 & $0.45 \pm 0.01$ & $0.45 \pm 0.01$ \\
\hline L8/L1 & $0.34 \pm 0.01$ & $0.35 \pm 0.01$ \\
\hline L9/L1 & $0.10 \pm 0.01$ & $0.10 \pm 0.00$ \\
\hline L10/L1 & $0.25 \pm 0.01$ & $0.25 \pm 0.01$ \\
\hline L11/L13 & $0.71 \pm 0.02$ & $0.71 \pm 0.01$ \\
\hline L12/L13 & $0.65 \pm 0.02$ & $0.66 \pm 0.01$ \\
\hline L14/L13 & $0.43 \pm 0.02$ & $0.45 \pm 0.02$ \\
\hline L15/L13 & $0.24 \pm 0.01$ & $0.24 \pm 0.01$ \\
\hline L16/L13 & $0.19 \pm 0.01$ & $0.19 \pm 0.02$ \\
\hline
\end{tabular}

Table (4): The morphometric data of the mandibles of some sheep and goat races. The unit of the mean values and the standard deviations are $\mathrm{cm}$. (Olopade and Onwuka 2005; Uddin et al., 2009, Advic et al., 2013)

\begin{tabular}{|c|c|c|c|c|c|}
\hline Parameters & Tuj & Morkaraman & Mehraban & WAD & Black \\
\hline L1 & 14.78 & 15.24 & 15.76 & 12.00 & 14.21 \\
\hline L10 & 3.64 & 3.72 & 3.98 & - & - \\
\hline L11 & 6.09 & 6.21 & 7.75 & 2.68 & 6.38 \\
\hline L13 & 8.54 & 8.7 & 9.57 & 6.90 & 8.83 \\
\hline
\end{tabular}




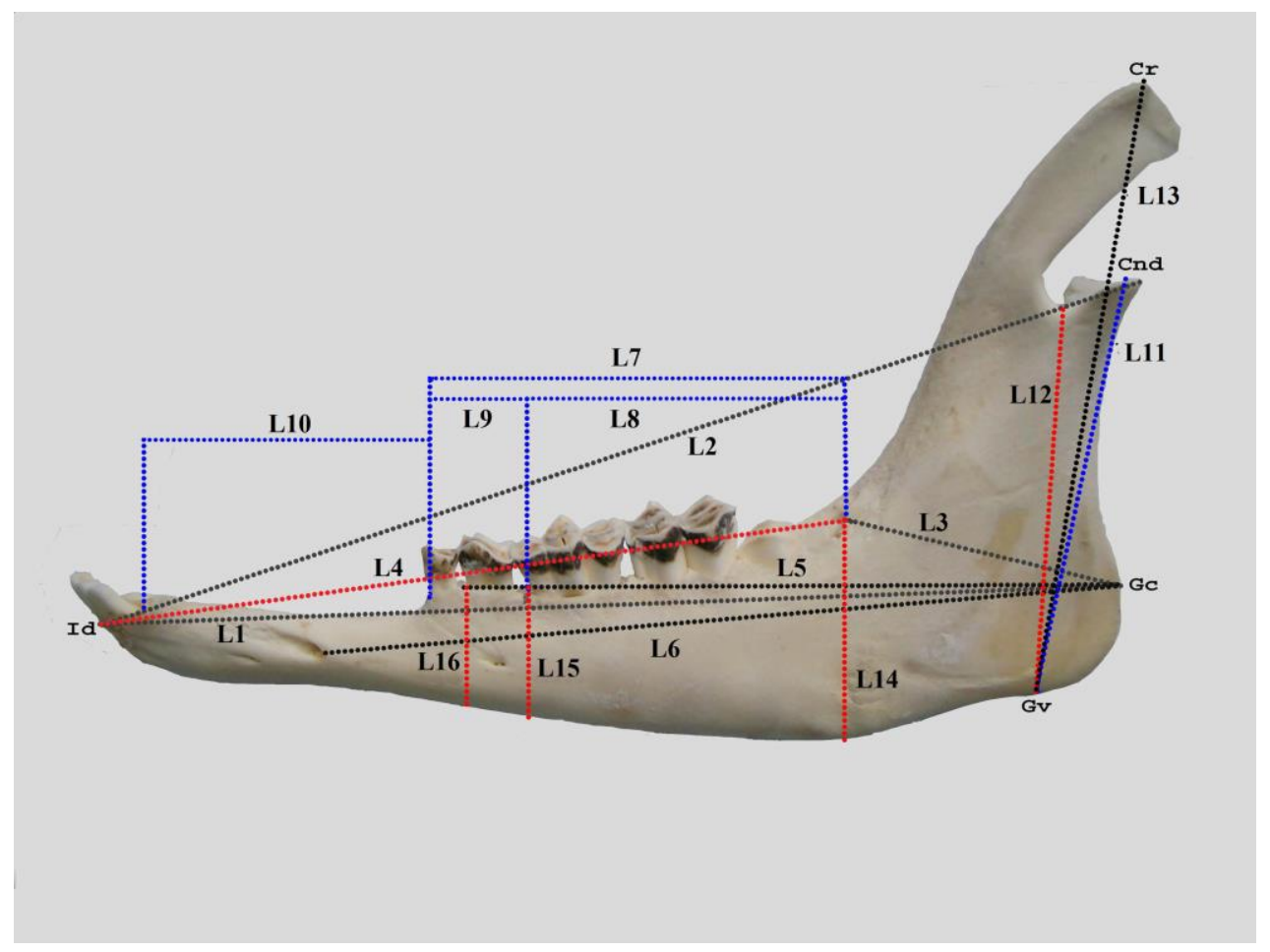

Fig (1): The length measurements of the mandible

Length 1(L1): lenght between gonion caudale and infradentale.

Length 2(L2): length between infra-dentale and aboral edge of condylar process.

Length $3(L 3)$ : length between gonion caudale and aboral alveolar edge of $3^{\text {rd }}$ molar tooth.

Length 4(L4): length between infra-dentale and aboral alveolar edge of $3^{\text {rd }}$ molar tooth.

Length 5(L5): length between gonion caudale and rostral alveolar edge of $2^{\text {nd }}$ premolar tooth.

Length 6(L6): length between gonion caudale and aboral edge of mental foramen.

Length $7(L 7)$ : length between first premolar tooth and last molar tooth.

Length 8(L8): length between first and last molar teeth.

Length 9(L9): length between first and last premolar teeth.

Length 10(L10): length of diestema.

Length 11(L11): length between gonion ventrale and condylion.

Length 12(L12): length between gonion ventrale and the deepest point of incisura mandibulae

Length 13(L13): length between gonion ventrale and coronion.

Length 14(L14): height of mandible level of alveolar edge of $3^{\text {rd }}$ molar tooth.

Length 15(L15): height of mandible level of rostral alveolar edge of $1^{\text {st }}$ molar tooth.

Length 16(L16): height of mandible level of rostral alveolar edge of $2^{\text {nd }}$ premolar tooth. 


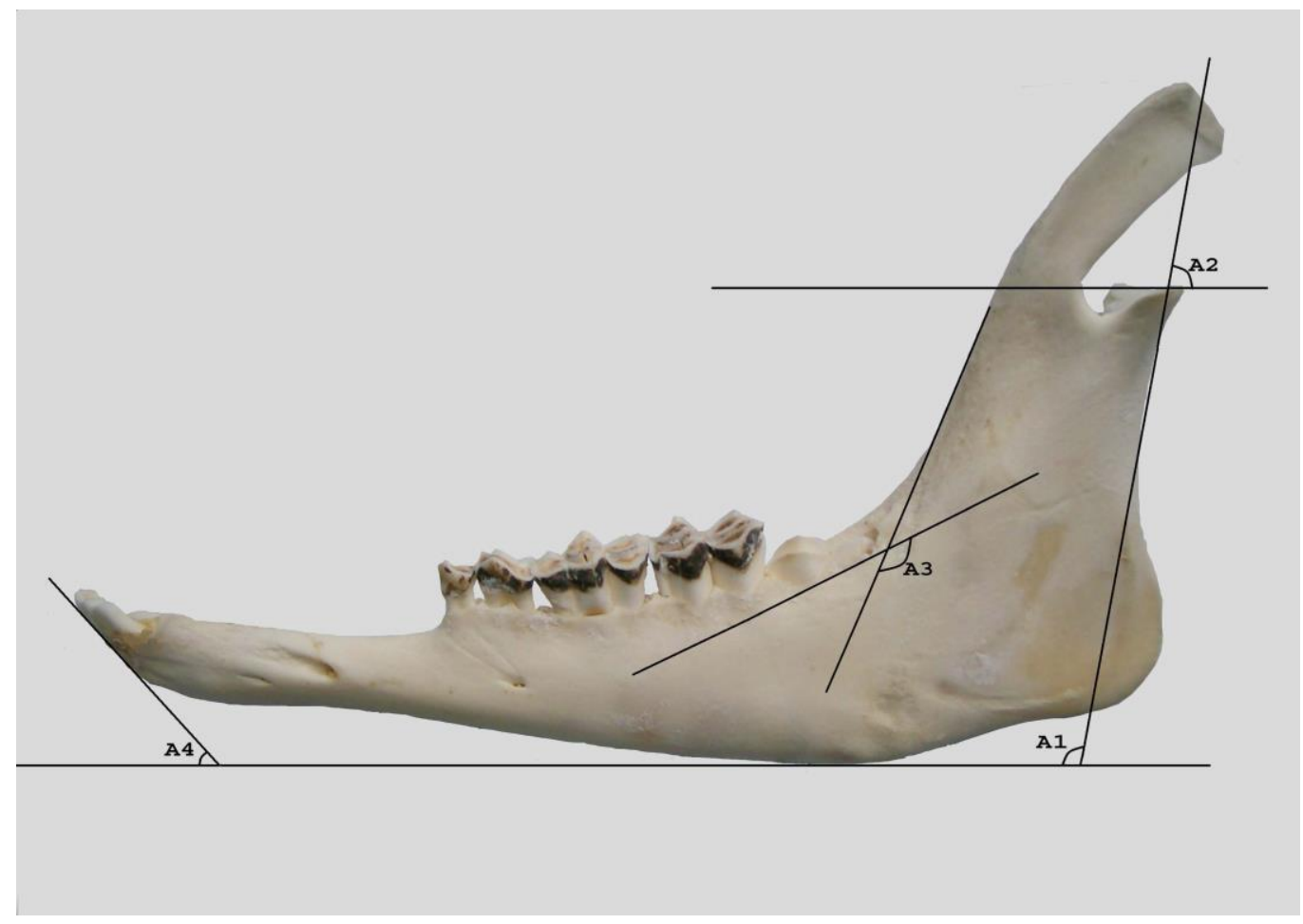

Fig (2): The angle measurements of the mandible

- $\quad$ Angle 1 (A1); angle between margo ventralis mandibulae and margo caudalis mandibulae.

- $\quad$ Angle 2 (A2); angle between tuber mandibulae of the condylar process and margo caudalis mandibulae.

- $\quad$ Angle 3 (A3); angle between ramus mandibulae and margo alveolaris of pars molaris corporis mandibulae

- $\quad$ Angle 4 (A4); angle made between rostral edge of the corpus mandibulae and the margo ventralis mandibulae.

* This study has been presented as a poster in VIII. National (International participation) Veterinary Anatomy Congress organized in Istanbul at 25-28 June 2013. 